\title{
The Present State of Fieldwork Education and the Problems of Psychiatric Occupational Therapy in South Korea
}

\author{
Eom Yoon-jeong1, Yamane Hiroshi, PhD² \\ ${ }^{1}$ Doctor course, Graduate School of Medicine, Kyoto University \\ 2 Graduate School of Medicine, Kyoto University
}

\begin{abstract}
Occupational therapy (OT) provided by psychiatric institutions across South Korea is typically conducted by mental health professionals rather than occupational therapists (OTR). There are few psychiatric OTRs in Korea, and none have studied OT through an official program. To identify possible remedies for this situation, we analyzed questionnaire surveys and interviews from 14 OTRs and compared our data to the current literature. We found that OT in Korean psychiatry departments was fraught with legal, educational, and clinical problems. There were no established minimum standards for OT education, OT was typically conducted by other mental health professionals, OTRs found it difficult to gain confidence, and students had not been given the necessary psychiatric fieldwork experience. Examining these results, we found a need for the revision of existing laws and enactment of better laws regarding OT, rationalization of treatment costs, verification of clinical effects, and reform of psychiatric OT training programs.
\end{abstract}

Key words: Occupational therapy, Fieldwork education, Psychiatric rehabilitation in South Korea

(Asian J Occup Ther 10: 9-16, 2014)

\section{Introduction}

The Mental Health Act of South Korea was amended in 2008 and executed from March 2009 onward. This act was the first law to define occupational therapy (OT) for hospitalized patients (Ministry of Health and Welfare, 2008) and the occupational therapist (OTR) as an agent of rehabilitation activities (Ministry of Health and Welfare, 2009). However, the therapy currently provided in psychiatry departments throughout Korea is implemented by social welfare workers, nurses, or other clinical psychologists; these are referred to as mental health agents, of which OTRs are not considered to be a part (Ministry of Health and Welfare, 2008; Ministry of Health and Welfare, 2010). The duties of mental health agents are, in general, focused on hospital cleaning, outdoor work, and occupational training for the rehabilitation of patients; this phenomenon has been noted to be problematic, such

Received: 2 July 2012, Accepted: 1 March 2013

Corresponding to: Eom Yoon-jeong, Graduate School of Medicine, Kyoto University, 53 Kawahara-cho, Shogoin, Sakyo-ku, Kyoto, Japan e-mail: oolleda@gmail.com

(C2014 Japanese Association of Occupational Therapists as group administration and exploitation of incomes in Seikatu-ryoho (Japanese) domains in Japan (Ministry of Health and Welfare, 2012a; Yoon-Jeong, E., \& Hiroshi, Y., 2011; Hiroshi, Y., 2010). No formal system for OT for the mentally ill has been established in Korea, unlike in Japan and throughout the West. While the number of OTRs in Japan reached 57,196 (association members: 44,942) in March 2012 (The Secretariat of the Japanese Association of Occupational Therapists, 2012), and the country had 7,637 psychiatric OTRs according to 2010 statistics (The Secretariat of the Japanese Association of Occupational Therapists, 2011), the number of OTRs in Korea reached only 6,446 in 2011 (The Secretariat of the Korean Association of Occupational Therapists, 2013), with only 20 psychiatric OTRs (Joo-Eon, L., 2011). Korea has few psychiatric OTRs, and they have not been formally trained in OT or psychiatric OT in an official program. Thus, psychiatric OTRs end up performing activities similar to those performed by mental health agents and the professionalism of psychiatric OT remains underdeveloped (Table 1).

In this study, we analyzed the problems related to the current conditions of psychiatric clinical education in South Korea and existing laws and training regulations 
Table 1. Comparison of mental health agent and agent of rehabilitation activities

\begin{tabular}{lll}
\hline & \multicolumn{1}{c}{ Qualifications or requirements } & \multicolumn{1}{c}{ Duties } \\
\hline Mental health agent & $\begin{array}{l}\text { Social welfare workers, nurses, clinical } \\
\text { psychologists }\end{array}$ & $\begin{array}{l}\text { In general, focused on hospital cleaning, outdoor work, } \\
\text { and occupational training for the rehabilitation of patients }\end{array}$ \\
\hline Agent of rehabilitation activities & $\begin{array}{l}\text { OTRs, social welfare workers, nurses, } \\
\text { and so on }\end{array}$ & $\begin{array}{l}\text { Psychiatric OTRs end up performing activities similar to } \\
\text { those performed by mental health agents }\end{array}$ \\
\hline
\end{tabular}

Table 2. Details of the questionnaire

1. How many students do you receive a year?

2. Please explain what aspects of training you place emphasis on when supervising and educating your students.

3. Please give your opinion of the current situation of psychiatric clinical training in Korea and any problems you encountered during your time as a supervisor of fieldwork education.

4. Do you currently lecture at a college or university? If yes, please write the name(s) of places where you lecture, and briefly explain how you feel about giving lectures on psychiatry.

in order to fully understand the task of developing and expanding psychiatric OT.

\section{Research Methods}

To analyze the current state of the clinical education of psychiatric OT in Korea, questionnaires were sent via e-mail to 14 OTRs working in psychiatry departments throughout Korea. We collected additional data by conducting personal interviews with the six OTRs that consented to participate. Table 2 presents the details of the self-administered questionnaire.

Furthermore, after comparing and analyzing the current literature and data regarding clinical training rules, laws, and institutions, we were able to review the problems that hinder OT in Korea on the basis of the interviews held with psychiatric OTRs.

\section{Results}

\section{Clinical training of psychiatric OT in Korea}

The questionnaire was sent to 14 OTRs working in the psychiatric departments of 12 institutions in Korea in 2010. Results are based on data from 10 respondents from 10 institutions; the collection rate was $71 \%$ (83\% per institution).

1) Questionnaire results

(1) Number of trainees per year

Results showed that five institutions (50\%) had received trainees in the past year. The number of students annually received in each institution ranged from 6 to 40 , with 89 students receiving training in psychiatry departments nationally. The remaining $50 \%$ of institutions did not administer OT training to their students.

(2) Emphasis on training guidance

Many subjects reported that they tended to place emphasis on teaching the most basic concepts to their trainees. Three subjects selected the following items: "explain the basic concepts of OT and the current status of the psychiatry department," "teach the necessity and role of OT in the psychiatry department," "pay attention to privacy and confidentiality issues," and "teach the basic terms, symptoms, and drugs related to various mental disorders." In contrast, two subjects selected the following items: "make sure trainees gain diverse experiences in the psychiatry department" and "pay attention to communication skills used with patients." In addition, two subjects mentioned students' lack of knowledge of the main concepts of psychiatry.

(3) Problems and opinions associated with psychiatric clinical training

"Students' lack of knowledge regarding psychiatric OT," "difficulty of carrying out clinical training with students who have not received a basic theoretical education," and "students' lack of understanding of psychiatric principles, as students are taught by faculty with little clinical experience" were selected by three subjects. This finding confirms that many OTRs were discontented with the low quality of the available training programs. Furthermore, subjects also mentioned problems related to human resources; three subjects selected "less time to teach students due to a busy clinical schedule," and two subjects selected, "lack of clinical training facilities" and "difficulties related to lack of adequate psychiatric OTRs." In addition, "outdated development of psychiatric OT," "absence of psychiatric OT supervisor," "communication problems," and "lack of places required for training, providing support, and exchanging information in relation to psychiatric OT" were each selected by two subjects.

(4) University lectures

Only one of the OTRs surveyed gave university lectures, having worked at two four-year colleges. This indicates that among the 25 four-year colleges nationwide, only two (8\%) provided a psychiatric OT curriculum in 
which students could learn from a psychiatric OTR with clinical experience. In addition, two of the surveyed OTRs had given lectures in the past, and one OTR occasionally gave special lectures.

(5) Views on the lectures

In their lectures, subjects generally acknowledged the lack of psychiatric OTRs in South Korea and the few employment opportunities available in psychiatric health. Other views included "the need to attract national attention to students' preferences for larger hospitals," "the tendency for students to show a greater interest in fields other than psychiatry," "the need for lectures provided by specialists with clinical experience," "the need for support from a professional association," "the need to establish formal OT concepts," and "the need for support, assistance, and interest in OT."

2) Interview results

In general, subjects were apprehensive about the future of psychiatric OT in Korea and requested the active support of the Korean Association of Occupational Therapists (KAOT) with regard to the legal and institutional problems that psychiatric OT must solve.

Subjects were largely dissatisfied with the fact that students without basic knowledge in clinical training had been sent to the psychiatry department, and they noted a strong need to recruit teachers with clinical experience. Because many students who attended psychiatric training sessions did not know even the most basic concepts related to mental disorders and general psychiatry, the first few weeks were generally spent on teaching basic theory. Furthermore, many subjects indicated that the absence of psychiatric OT supervisors led to hospitals presenting different OT content, making it difficult to have confidence in currently implemented therapy throughout the country.

\section{Comparison with clinical training laws in various countries}

Clinical training of more than 1,000 hours is expected to meet the minimum educational standards for OTRs according to the World Federation of Occupational Therapists (WFOT) (World Federation of Occupational Therapists, 2002), which have become the standards for OT training. Furthermore, students must have two months of a clinical training, and supervisors, at least one year of experience. Although the standards do not state the time or specifics of the arrangement for clinical training in psychiatry, they do mention the following: "Students experience a range of different fieldwork placements that require them to integrate knowledge, skills, and attitudes to practice with a range of people with different needs and in different circumstances" (World Federation of Occupational Therapists, 2002). The reason for avoiding any detailed requirements is that the standards in the 2002 re- vised version (World Federation of Occupational Therapists, 2002) were created to allow flexibility in curriculum content and to relax clinical training requirements. As the standards were written in consideration of national characteristics, rules differ according to country (Table 2).

The U.S. clinical training rules present precise regulations, qualifications, purposes, and roles for all of the following: Level I/Level fieldwork, OTR/Occupational Therapy Assistant (OTA), doctoral/master's degree levels, and student/fieldwork educators (FWE)/academic fieldwork coordinators (AFWC)/program faculty. While they do not present specific rules for clinical training in psychiatry, their specifications for Level II fieldwork state the following: "It is recommended that the student be exposed to a variety of clients across the life span and to a variety of settings." Furthermore, they also specify that Level II fieldwork must last at least 24 weeks, must be implemented in 1-4 institutions, and supervision must be provided by an OTR or OT with at least one year of experience (American Occupational Therapy Association, 2010; American Occupational Therapy Association, 2009).

Like the U.S., Canada does not present specific rules regarding psychiatric clinical training. However, it requires university fieldwork coordinators/professors to "coordinate offers and requests for placements and whenever possible match students and sites according to students' academic and fieldwork profiles and interests" (Committee on University Fieldwork Education, 2011). In addition, detailed regulations are stated in their academic accreditation indicators (Canadian Association of Occupational Therapists, 2010) (Table 3).

In contrast, Ireland presents the following detailed regulations in addition to the WFOT standards: a minimum of 250 hours of training in psychiatry and a minimum of 250 hours of training in body and sensory disorders (Association of Occupational Therapists of Ireland, 2013).

Japan adopted the rules of the Japanese Association of Occupational Therapists (JAOT) and the Ordinance of the Ministry of Health, Labour and Welfare. Although the designated rules of the Ministry recommend 810 hours of clinical training (two-thirds of which must be conducted in hospitals and medical centers), the JAOT designates the minimum as 1,000 hours in accordance with the minimum standards of the WFOT. Furthermore, both the Ordinance of the Ministry of Health, Labour and Welfare and the JAOT designate a clinical educator as a person who has accumulated at least three years of clinical experience after acquiring a license, and they indicate the need to carry out training regardless of disorder, stage, or age (Japanese Association of Occupational Therapists Education Division, 2003; Ministry of Education, Culture, 
Table 3. Minimum standards for psychiatric OT fieldwork education in several countries

\begin{tabular}{|c|c|c|c|c|c|c|}
\hline & WFOT & USA* & Canada & Ireland & Japan & Korea \\
\hline $\begin{array}{l}\text { Minimum fieldwork } \\
\text { hours and duration }\end{array}$ & 1,000 hours & 24 weeks & 1,000 hours & 1,000 hours & $\begin{array}{l}\text { JAOT: about 1,000 } \\
\text { hours Government: } \\
810 \text { hours }\end{array}$ & None \\
\hline $\begin{array}{l}\text { Minimum fieldwork } \\
\text { hours in psychiatric } \\
\text { care }\end{array}$ & $\begin{array}{l}\text { No particular amount } \\
\text { (a range of different } \\
\text { fieldwork place- } \\
\text { ments) }\end{array}$ & $\begin{array}{l}\text { No particular amount } \\
\text { (students should be } \\
\text { exposed to a variety } \\
\text { of settings) }\end{array}$ & $\begin{array}{l}\text { No particular amount } \\
\text { (match students and } \\
\text { sites according to } \\
\text { students' interests) }\end{array}$ & 250 hours & No particular amount & None \\
\hline Fieldwork placements & $\begin{array}{l}\text { A range of different } \\
\text { fieldwork placements }\end{array}$ & 1-4 institutions & $\begin{array}{l}\text { All fieldwork occurs } \\
\text { in approved sites }\end{array}$ & Same as WFOT & $\begin{array}{l}\text { More than two-thirds } \\
\text { is at hospitals or } \\
\text { clinics }\end{array}$ & None \\
\hline $\begin{array}{l}\text { Supervisor (minimum } \\
\text { fieldwork experience) }\end{array}$ & $\begin{array}{l}\text { OTR or OT educator } \\
\text { (1 year) }\end{array}$ & $\begin{array}{l}\text { OTR (none) } \\
\text { OTA (1 year) }\end{array}$ & OTR (1 year) & Same as WFOT & OTR (3 years) & None \\
\hline
\end{tabular}

Table 4. Occupational qualifications including OTRs

Occupations and qualifications

\begin{tabular}{|c|c|}
\hline $\begin{array}{l}\text { Rehabilitation activity } \\
\text { personnel }\end{array}$ & $\begin{array}{l}\text { 1) Social welfare workers, nurses, and OTRs. } \\
\text { 2) Any person who has graduated from the Department of Psychology in a university which conforms to } \\
\text { Article Two of the Higher Education Act. } \\
\text { 3) Any person who has taken courses related to psychiatric rehabilitation activities at a college for three or } \\
\text { more years and has graduated. }\end{array}$ \\
\hline Job coaches & OTRs, social welfare workers, nurses, nurse assistants \\
\hline
\end{tabular}

Sports, Science and Technology of Japan, 2010; Ministry of Health, Labour and Welfare of Japan, 1999; Ministry of Education, Culture, Sports, Science and Technology of Japan, 1976). In Korea, OTR training standards have not been designated by either the KAOT or the government.

Furthermore, while Japanese WFOT standards have approved 132 out of 183 schools (72\%) (The Secretariat of the Japanese Association of Occupational Therapists, 2013) as suitable for training, Korea has only approved 8 out of 55 schools $(15 \%)$ (The Secretariat of the Korean Association of Occupational Therapists, 2013; The Secretariat of the World Federation of Occupational Therapy, 2013), including universities and junior colleges in 2013. Maximum number of incoming students accepted is 1,985 in 2013 (The Secretarit of the Korean Association of Occupational Therapists, 2013).

\section{Rehabilitation in the field of psychiatry in Korea}

Unlike other countries, Korea provides psychiatric rehabilitation under the "mental health agent system." This system was first introduced when the need for the rehabilitation and social integration of the mentally ill was raised by the enactment of the Mental Health Act in 1995. This converted the law from a policy focused on hospitalization and isolation to a policy focused on rehabilitation and social return.

As the development of specialists in various fields was required by this process, Korea introduced the mental health agent system in March 1997. Under this system, training institutions designated by the Ministry of Health and Welfare recruit trainees once a year to begin training programs during March and September. Programs are classified into Level 1 and Level 2 programs. The Level 2 program requires at least one year of training, whereas the Level 1 program requires at least three years. However, qualifications vary, as in some cases students are permitted to apply for the Level 1 program after gaining five years of clinical experience since acquiring the Level 2 certificate. After completing the training program, subjects can apply for a certificate from the Ministry of Health and Welfare and thereby become an official mental health agent.

The number of mental health agents reached 9,947 in December 2009, and nearly 1,000 students acquire the certificate every year (Ministry of Health and Welfare, 2010). However, this system is restricted to nurses, social welfare workers, and clinical psychologists; OTRs are affiliated with rehabilitation activity personnel and job coaches, as shown in Table 4 (Ministry of Health and Welfare, 2009; Ministry of Health and Welfare, 2010; Ministry of Health and Welfare, 2012b).

Thus, OT was first implemented in Korea by individuals in other occupations rather than by OTRs. Although OT can be implemented by either mental health agents or OTRs according to a psychiatrist's direction, in cases where the patient has legally requested and consented to treatment, OT is generally implemented by job coaches (OTRs, social welfare workers, nurses, and nurse assis- 
Table 5. Sections of the Korean Mental Health Act relevant to OT

Article 46-2 (Occupational therapy in mental hospitals)

1. If it is judged that simple work such as making crafts may be helpful for the treatment of a hospitalized patient or the social reinstatement of a hospitalized patient, the chief of the psychiatric institution may instruct them to do such work when it presents no risk to their health.

2. The occupation in Section 1 will be enforced only with application or consent of the person and in accordance with the psychiatrist's instructions. In the case of mental recreation facilities, however, mental health professionals may direct the specific method of the occupation under the guidance of a psychiatrist.

3. If the occupation is instructed as per Sections 1 or 2, the chief of the psychiatric institution must record the details in the medical chart or occupational therapy record of the patient or inmate.

4. Specific matters of Section 1 such as occupation time, risk, and place will be decided by the Ministry of Health, Welfare and Family. [Enactment of this article 2008.3.21] [Date of enforcement 2009.3.22]

Table 6. Sections of the Enforcement Regulations of the Mental Health Act relevant to OT

Article 23-2 (Occupational therapy for hospitalized patients)

1. If the occupation is conducted at a psychiatric institution as per Section 1 of Article 46-2, the total hours must not exceed 6 hours a day or 30 hours a week. If the occupation is conducted outside, instead of at the psychiatric institution, the total hours must not exceed 8 hours a day or 40 hours a week. Moreover, the occupation must be conducted in a place with facilities equipped appropriately for the occupation, for example, an occupational rehabilitation training room.

2. The occupation in Section 1 will be conducted with the consent or application of the patient and carried out under the instruction of a psychiatrist. However, the occupation must be conducted in a safe environment by hiring a mental health professional or occupational therapist, and tools that may harm mental patients or others, such as scissors or cutters, must be monitored and used safely.

3. If the occupation brings in money, as per Section 1, the amount will be calculated by subtracting the charges of the occupation, for example, the purchase of raw materials, and it must be paid directly to the bank account of the hospitalized patient.

[Enactment of this article 2009.3.20]

Table 7. Related matters of occupational therapy and social rehabilitation training in nursing facilities

1. The chief of the psychiatric institution may instruct hospitalized patients to do simple occupations, such as making envelops, cleaning, cooking, and washing of the hospital where patients can be admitted, in consideration of their health and the types/hours/dangers/places of that occupation, for the purpose of rehabilitation training.

2. The occupation in Section 1 must not exceed 6 hours a day or 30 hours a week. (If the occupation is conducted outside the nursing facilities, the total hours must not exceed 8 hours a day or 40 hours a week, and the occupation must be conducted in a place with facilities equipped appropriately for the occupation, e.g., an occupational rehabilitation training room.)

3. The occupation in Section 1 will be enforced with the consent or application of the patient and carried out under the instruction of a psychiatrist or mental health professionals under the guidance of a psychiatrist. However, occupation therapists, social workers, nurses, and assistant nurses will be used as vocational training instructors, and hospitalized patients must be protected by providing the occupation in a safe environment. Vocational training instructors must make a record of the occupational therapy specified in appended form 15 , and must receive further confirmation from a psychiatrist.

Table 8. Medical expenses of health insurance relating to OT

\begin{tabular}{|c|c|c|c|}
\hline Class No. & Code & Classification & Points \\
\hline \multirow{5}{*}{ 아-4 } & NN040 & Occupational or recreation therapy [music, painting, calligraphy, sculpture, sports, etc.] & 54.15 \\
\hline & & Notes: & \\
\hline & & 1. Stated points will be calculated regardless of the number of treatments carried out. & \\
\hline & & $\begin{array}{l}\text { 2. For outpatients, points will be calculated once a week, and for hospitalized patients, } \\
\text { points will be calculated five times a week. }\end{array}$ & \\
\hline & & 3. The price for raw material will not be calculated separately. & \\
\hline
\end{tabular}

tants) under the guidance of mental health agents who are directly supervised by a psychiatrist (Ministry of Health and Welfare, 2008; Ministry of Health and Welfare, 2009; Ministry of Health and Welfare, 2012a). Tables 5-7 present these details.
As OTRs are not currently affiliated with mental health agents, "Occupational or Recreation Therapy" is the only health insurance item that can be requested (Ministry of Health and Welfare, 2012b), and as $66 \mathrm{KRW}$ is paid per point (unit cost of hospitals, nursing hospitals, 
Table 9. Comparison of medical treatment fees related to psychiatric OT between Japan and Korea

\begin{tabular}{|c|c|c|c|c|}
\hline Country & Class & Points & W/¥ & US\$ \\
\hline Korea & $\begin{array}{l}\text { Occupational or recreation therapy } \\
\text { [music, painting, calligraphy, sculpture, sports, etc.] } 6 \text { hours a day, } 30 \text { hours a week }\end{array}$ & 54.15 & 3,574 & 3.13 \\
\hline \multirow{14}{*}{ Japan } & Psychiatric occupational therapy, 2 hours once a day & 220 & 2,200 & 27.57 \\
\hline & Social skills training therapy at the hospital (within 6 months after admission), once a week & 100 & 1,000 & 12.53 \\
\hline & Social skills training therapy at the hospital (over 6 months after admission), once a week & 75 & 750 & 9.40 \\
\hline & Short-stay psychiatric care (small scale), 3 hours a day & 275 & 2,750 & 34.46 \\
\hline & Short-stay psychiatric care (large scale), 3 hours a day & 330 & 3,300 & 41.35 \\
\hline & Psychiatric day care (small scale), 6 hours a day & 590 & 5,900 & 73.93 \\
\hline & Psychiatric day care (large scale), 6 hours a day & 700 & 7,000 & 87.72 \\
\hline & Psychiatric night care, 4 hours a day, after $16: 00$ & 540 & 5,400 & 67.67 \\
\hline & Psychiatric day/night care, 10 hours a day & 1040 & 10,400 & 130.33 \\
\hline & Psychiatric discharge guidance fee, once during hospitalization & 320 & 3,200 & 40.10 \\
\hline & $\begin{array}{l}\text { Psychiatric home visit nursing guidance fee (I), } 3 \text { times a week } \\
\text { (within } 3 \text { months after discharge is } 5 \text { times a week) }\end{array}$ & 575 & 5,700 & 71.43 \\
\hline & Psychiatric home visit guidance (before discharge) fee, 3 times during hospitalization & 380 & 3,800 & 47.62 \\
\hline & Severe dementia day care fee, once per day & 1040 & 10,400 & 130.33 \\
\hline & Addition of environmental improvement for life with medical treatment & 40 & 400 & 5.01 \\
\hline
\end{tabular}

* Exchange rate: reference date is 2012.03.09 (W/US\$: 1,140, ¥/US\$: 79.77).

and general hospitals) (Ministry of Health and Welfare, 2012b), the cost amounts to about 3,574 KRW (Table 8). This is extremely low when compared with the number of health insurance items related to OT and their cost in Japan (Japanese Association of Occupational Therapists, 2010). Table 9 compares the status of OT in Korea with that of Japan.

\section{Review}

As the survey and interviews were conducted with only psychiatric OTRs, this study is limited in that the problems indicated in the research are based on the perspectives of OTRs only. However, the results indicate that the current state of clinical training for psychiatric OTs in Korea is complicated and problematic. The causes of these problems were observed to be related to the lack of clinical training institutions and training programs and legal, institutional, and regulations-related problems.

1) Clinical training institutions

Although the questionnaire was carried out in 2010, there have been no significant changes in the psychiatric OT training situation since that time. The number of psychiatric OTRs remains currently at about 20 (2012). Thus, when considering that the number of freshmen enrolled in universities and junior colleges was 1,870 in 2011, students who graduated with psychiatric clinical experience account for only $5 \%$ of the total number of students. The remaining $95 \%$ graduated without receiving any sort of experience or training in psychiatric practice. The reason for the scarcity of clinical training institutions in Korea is considered related to the low position relegated to OTRs in psychiatry and their overall lack of confidence. Consequently, OTRs do not want to accept students or reveal their skills and position to trainees.
This study first examined the position of OT and OTRs in psychiatry departments across Korea. As OTRs are affiliated with rehabilitation activity personnel or job coaches rather than mental health agents, they do not take a leading role in treating patients. Instead, they focus more on assisting with therapy or reporting on the overall safety of patients. Furthermore, the fact that it is more cost-effective to hire mental health agents also serves as an obstacle to increasing the number of clinical training institutions. In addition, $50 \%$ of the institutions surveyed did not receive trainees at all, even though they had employed OTRs in their psychiatry departments. This is probably because although there are many OTRs working, they might be anxious and doubtful about the therapy itself, as they themselves have never received in-depth training in psychiatric OT. In addition, many institutions do not receive trainees because they have begun operating only recently and thus cannot afford it.

2) Training programs

With regard to psychiatric OT training in universities, many subjects expressed strong discontentment with the fact that students must learn the basics during their training program due to a lack of knowledge in psychiatric OT caused by inadequate training, theory-based curriculums, and a lack of professors with sufficient clinical experience in psychiatric care. Thus, it is important for South Korea to increase the number of college professors teaching psychiatric OT and to improve the quality of their teaching. To do this, the KAOT must establish minimum training standards and take the time to foster teachers who can properly teach students the basics of psychiatric OT before graduation. Furthermore, the demand for OTRs is low, and it is difficult for OTRs to gain confidence in practice. They tend to have trouble obtaining appropriate medical fees in clinical situations, as they 


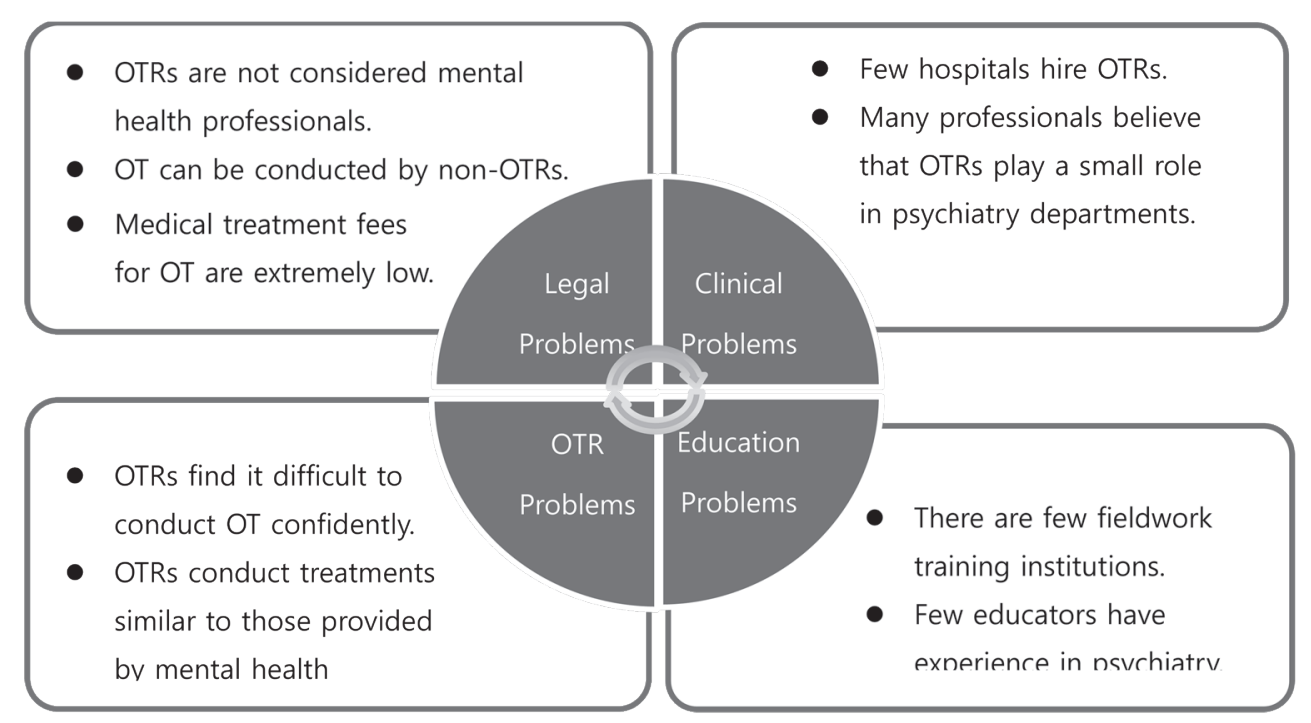

Fig. 1. Major problems in psychiatric OT in Korea

have a somewhat ambiguous position within the hospital. In order to improve this situation, it is important to help OTRs gain confidence in their abilities and the care they provide, enhance the overall quality of OT offered in hospitals, and emphasize the advantages of OTRs above other medical staff in administering OT. Furthermore, it is essential to recruit supervisors responsible for mentoring future psychiatric OTRs. The KAOT must plan and eventually implement a more appropriate training program for OTRs who specialize in psychiatry.

3) Legal/institutional/designated regulations

Both the health insurance system and the Mental Health Act of Korea have allowed non-OTRs to conduct OT, thus giving it a low treatment cost and an insignificant position in care facilities. Although there is a fundamental problem in the development and dissemination of psychiatric OT in Korea, the quality of psychiatric OTRs has also been viewed as a significant problem from a legal perspective. The therapy currently administered in psychiatry departments throughout Korea is conducted primarily by mental health agents. To acknowledge the merits of using OTRs to conduct psychiatric OT, researchers must emphasize how the characteristics and advantages differ from the conventional methods with regard to the therapy's structure, content, and effects.

Furthermore, another significant problem is the absence of formal standards for OT training in Korea. As different standards have been designated for different OT training programs in other countries, including differences in teaching qualifications, curriculum, and clinical training time, the quality of such programs varies significantly throughout Korea. Furthermore, because of the small number of hospitals that have implemented clinical training in their psychiatry departments, it is impossible for all students to receive practical psychiatric experience. It remains necessary to increase the overall quality of graduate students and OTRs by providing better psychiatric OT training, enacting official OT training standards by law, establishing minimum training requirements for the association, maintaining a consistent level of training across schools, and making it mandatory for all schools to provide basic psychiatric OT education.

\section{Conclusion}

As psychiatric OT in Korea is fraught with legal, educational, and clinical problems (Figure 1), it is difficult to present clear solutions. Although many areas must be improved, such as revising and enacting laws related to OT, rationalizing treatment costs, and verifying the clinical effects of OT, it is essential to reform psychiatric OTR training programs on a national level. Before the legal defects, problems of treatment costs, and inadequate clinical environments can be discussed, it is important to publicize the necessity for psychiatric OTRs through the practice of quality psychiatric OT; this would help establish OTR as an essential occupation in the field of psychiatry in the near future.

\section{References}

American Occupational Therapy Association (2010). Accreditation Council for Occupational Therapy Education (ACOTE®) standards and interpretive guidelines. American Occupational Therapy Association.

American Occupational Therapy Association (2009). Recommendations for occupational therapy fieldwork experiences. American Occupational Therapy Association.

Association of Occupational Therapists of Ireland (2013, Feb 
25), available from http://www.medicine.tcd.ie/occupational-therapy/practice-education/docs/Minimum-Standards-for-Practice-Education-in-Ireland.pdf.

Canadian Association of Occupational Therapists (2010). CAOT academic accreditation standards and self-study guide. Canadian Association of Occupational Therapists.

Committee on University Fieldwork Education (2011). Canadian guidelines for fieldwork education in occupational therapy. Association of Canadian Occupational Therapy University Program.

Hiroshi, Y. (2010). The mental disorders and occupational therapy. (3rd ed.). Tokyo (in Japanese).

Japanese Association of Occupational Therapists (2010). Guidance of medical insurance, care insurance, supports for persons with disabilities system of occupational therapy. Japanese Association of Occupational Therapists (in Japanese).

Japanese Association of Occupational Therapists Education Division (2003). Minimum standards for the education of occupational therapists. Japanese Association of Occupational Therapists (in Japanese).

Joo-Eon, L. (2011). The present state of the occupational therapists is somewhat changed. Korean Institution of Mental Health Occupational Therapy. http://cafe.naver.com/otmentalhealth/(accessed 2012-02-18).

Ministry of Education, Culture, Sports, Science and Technology of Japan (1976). Standards for establishment of special training colleges. Ministry of Education, Culture, Sports, Science and Technology (in Japanese).

Ministry of Education, Culture, Sports, Science and Technology of Japan (2010). Ministry of Health, Labour and Welfare: Designated provisions of training facility of physical therapists and occupational therapists school. Ministry of Education, Culture, Sports, Science and Technology (in Japanese).

Ministry of Health, Labour and Welfare of Japan (1999). Regarding training facility guidelines of physical therapists and occupational therapists. Ministry of Health, Labour and Welfare (in Japanese).

Ministry of Health and Welfare (2008). Mental health law. Ministry of Health and Welfare, Seoul (in Korean).

Ministry of Health and Welfare (2009). Enforcement regulations of mental health law. Ministry of Health and Welfare,
Seoul (in Korean).

Ministry of Health and Welfare (2010). The managerial guidance of mental health professionals system. Ministry of Health and Welfare, Seoul (in Korean).

Ministry of Health and Welfare (2012a). The guidance of mental health operations. Ministry of Health and Welfare, Seoul (in Korean).

Ministry of Health and Welfare (2012b). The medical expenses to be paid among employees' (monthly) salaries for the recovery of their diseases in health insurance. Ministry of Health and Welfare (in Korean).

The Secretariat of the Japanese Association of Occupational Therapists (2011). Statistics materials of Japanese Association of Occupational Therapists members in 2010. Occupational Therapy 30, 496-516 (in Japanese).

The Secretariat of the Japanese Association of Occupational Therapists (2012). Occupational therapists as of March 1, 2012. Japanese Association of Occupational Therapists News 362, 24 (in Japanese).

The Secretariat of the Japanese Association of Occupational Therapists (2013). A List of the national occupational therapists schools. Japanese Association of Occupational Therapists. http://www.jaot.or.jp/wp/members/ members2/2013school/(accessed 2013-02-25).

The Secretariat of the World Federation of Occupational Therapists (2013). Entry level Educational Programmes WFOT Approved. World Federation of Occupational Therapists. http://www.wfot.org/Education/Entrylevel Educational Programmes WFOT Approved. aspx (accessed 2013-0225).

The Secretariat of the Korean Association of Occupational Therapists (2013). The present state of the established schools. Korean Association of Occupational Therapists. http://www.kaot.org/(accessed 2013-02-25).

World Federation of Occupational Therapists (2002). Revised minimum standards for the education of occupational therapists. The Council of the World Federation of Occupational Therapists.

Yoon-Jeong, E., \& Hiroshi, Y. (2011). The current state of Korean psychiatric occupational therapy. The Journal of the Japanese Association of Occupational Therapists. 30, 137-146. (in Japanese). 